\title{
Generation of 2-Azapentadienyl Anions and Their Cycloaddition with Alkenes. Synthesis of 2-Alkenylpyrrolidines
}

\author{
William H. Pearson* and Valerie A. Jacobs
}

Department of Chemistry, The University of Michigan, Ann Arbor, MI 48109-1055

\begin{abstract}
Unsaturated imines 2 bearing an $N$-[1-(tri- $n$-butylstannyl)]alkyl group were transmetalated with $n$-BuLi to generate 2-azapentadienyl anions 3 which underwent [4 $\pi s+2 \pi s$ ] anionic cycloadditions with alkenes to affond 2-alkenylpyrmolidines 4 after workup with an electrophile. The alkenyl group could be oxidized to a diol, aldehyde, or ester. The imine 2 was found to undergo a [1,5]-sigmatropic rearrangement of the uri-nbutylstannyl group at $80^{\circ} \mathrm{C}$ to provide the 2 -azabutadiene 5 .
\end{abstract}

We have previously described the synthesis of pyrrolidines by the $[4 \pi s+2 \pi s]$ cycloaddition of nonstabilized 2-azaallyl anions with electron-rich alkenes, $, 1,2$ and we have recently extended the method to the synthesis of 1-pyrrolines and pyrroles. ${ }^{3}$ The anions are prepared by tin-lithium exchange of 2 (azaallyl)stannanes. We now wish to describe the generation and cycloaddition chemistry of 2-azapentadienyl anions 3 which allow access to 2-alkenylpyrrolidines 4 (Scheme 1). In conjunction with oxidative transformations, these pyrrolidines are potentially valuable synthetic intermediates for the preparation of biologically important pyrrolidines bearing hydroxyalkyl, aldehyde, and carboxylic acid functionality at $\mathbf{C}(2)$. While similar pyrrolidines may be accessed using dipolar cycloaddition reactions of carboxyl-bearing azomethine ylides, ${ }^{4,5}$ the two methods are complementary since azomethine ylide cycloadditions generally require electron-poor alkenes while non-stabilized 2-azaallyl anions are most efficient with electron-rich alkenes.<smiles>CCCCC(N)C(C)C</smiles><smiles>CC=CC=CC(=O)OCC(=O)OCC</smiles><smiles>C/C=C/C=N/C(C(C)C)C(CC)CCCC</smiles>
1.2 eq. n-BuLi 2 eq. alkene $-78^{\circ} \mathrm{C}, 15 \mathrm{~min}$ then $\mathrm{El}^{+}$<smiles>CC=CC=NCC(C)C</smiles>

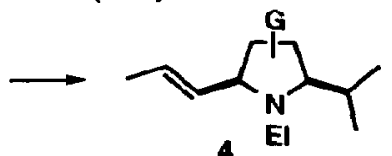

Schome 1. Generation and Cyclaaddition of 2-Azapentadienyl Anions

A number of studies on 2-azapentadienyl anions have appeared. ${ }^{6-8}$ In every case, the anions were generated by deprotonation of imines, particularly those made by the condensation of allylic amines with aromatic aldehydes and ketones. As we have observed, this approach is limited to non-enolizable imines. ${ }^{1}$ The tin-lithium exchange approach described below is not subject to this limitation. Spectroscopic and computational studies on 2-azapentadienyl anions have revealed that 1-phenyl-2-azapentadienyl anions prefer the all "W" conformation (i.e., all s-trans), whereas s-cis conformations are also present in 1-alkyl-2azapentadienyl anions. ${ }^{7}$ Regarding the chemistry of 2-azapentadienyl anions, 1,5-electrocyclization to 1pyrrolines has been found to occur at temperatures above $0^{\circ} \mathrm{C} .{ }^{6}$ Similar electrocyclizations have been observed for alkenyl azomethine ylides. ${ }^{9,10}$ Other chemistry of 2-azapentadienyl anions includes alkylation, addition to aldehydes, conjugate addition, and in some cases cyclization with enones to give pyrrolidines. 7,8 The latter are thought to proceed by conjugate addition followed by cyclization rather than by a concerted cycloaddition. We have now found that simple alkyl-substituted 2-azapentadienyl anions may be prepared by 
tin-lithium exchange. These anions undergo cycloadditions with electron-rich alkenes by what is presumed to be a concerted process.

Condensation of the $\alpha$-amino stannane $1^{11,12}$ with crotonaldehyde afforded the stannyl imine 2 in excellent yield (Scheme 1). Purification was not only unnecessary, but is inadvisable since attempted distillation afforded the new imine 5 by a [1,5]-sigmatropic rearrangement of the tributylstannyl group (eq. 1). This rearrangement could also be observed by ${ }^{1} \mathrm{H}$ NMR spectroscopy at $80^{\circ} \mathrm{C}$ in a sealed tube. First-order kinetics were observed $\left(k_{1}=3.5 \times 10^{-5} \mathrm{sec}^{-1} ; t_{1 / 2}=328 \mathrm{~min}\right.$ at $\left.80^{\circ} \mathrm{C}\right)$. The interconversion of the $E$ - and $Z$ forms of $\mathbf{2}$ is presumed to be much faster than the sigmatropic rearrangement. Only one geometrical isomer of 2 (assumed to be the $E$-isomer) was observed by NMR. Similar [1,5]-tin shifts have been observed in 5stannyl-1,3-pentadienes. ${ }^{13}$ The greater thermodynamic stability of the 2-azabutadiene 5 versus the 1azabutadiene 2 is expected. 6

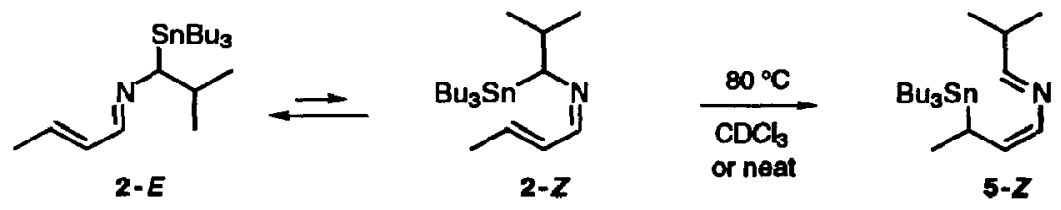

Cycloadditions were performed by mixing the stannane 2 with two equivalents of the anionophile in a small amount of THF, then adding this mixture to 1.2 equivalents of $n-B u L i$ (a 2.1M solution in hexane) in THF at $-78^{\circ} \mathrm{C}$ (final anion concentration ca. $0.1 \mathrm{M}$ ). After $15 \mathrm{~min}$, the mixture was quenched with either water or two equivalents of $\mathrm{MeI}$ or $\mathrm{MeO}_{2} \mathrm{CCl}$. Normal aqueous workup provided the 2-alkenylpyrrolidines 6-14 (Table 1, entries 1-5). The pyrrolidines were formed as a mixture of regio- and stereoisomers in most cases, although the 1,3-cis relationship of the groups at $\mathrm{C}(2)$ and $\mathrm{C}(5)$ is consistently observed as would by expected from cycloaddition of the "W" form of the anion 3. Such stereoselectivity is consistent with our prior work on the cycloaddition of 1,3-disubstituted 2-azaallyl anions. $1 \mathrm{~b}$ Also consistent is the failure of these anions to undergo cycloaddition with simple alkenes such as norbornene to provide adducts 15 (entry 6 ). 1 Stilbene and $\alpha$-methyl styrene are successful anionophiles, providing aryl-substituted pyrrolidines 6-11 (entries 1-3). The trans-geometry of stilbene is translated into a 3,4-trans relationship in the pyrrolidines 6-9 (entries 1 and 2). In order to prepare pyrrolidines bearing no aryl groups, the use of phenyl vinyl sulfide as the anionophile is a useful solution since we have previously shown that the phenylthio group may be removed reductively or oxidatively. ${ }^{\text {lc }}$ Entries 3 and 4 illustrate the formation of phenylthio-substituted pyrrolidines 12-14.

Table 1. Generation and Cycloaddition of 2-Azaallypentadienyl Anion 3.

Entry Anionophile Electrophile


Table 1 (continued)

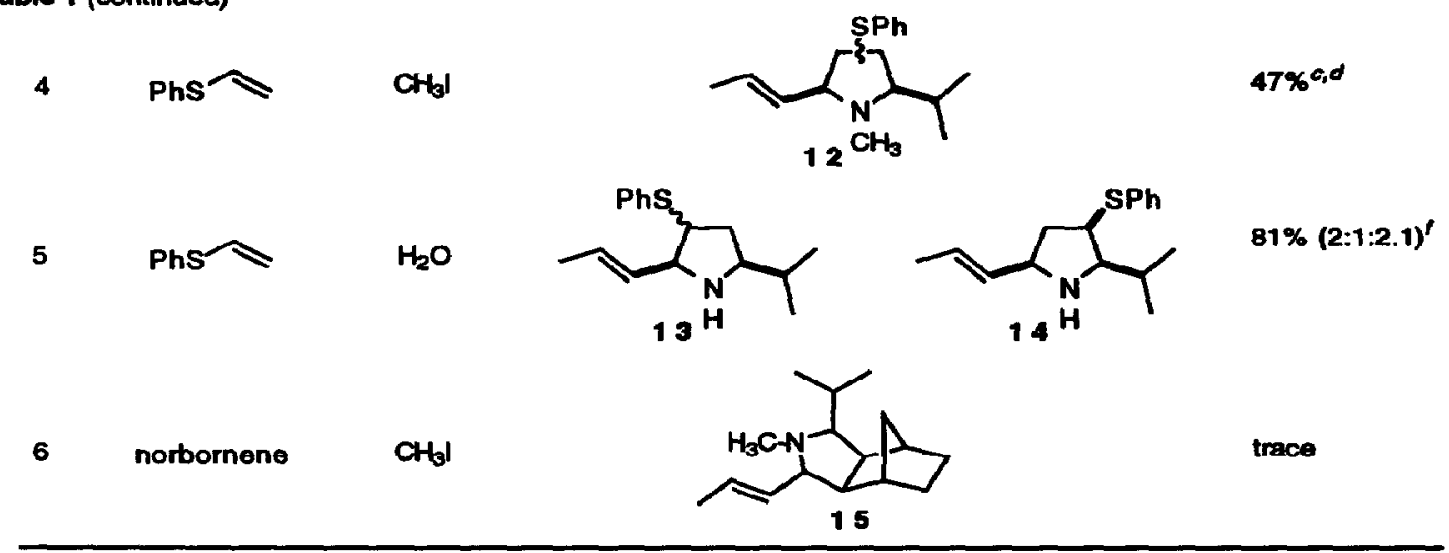

(a) Isolated, chromatographed yields. (b) lsomers separable. (c) Isomers not separable. (d) Assignment of regioand stereochemistry was not possible. (e) ca. equal amounts of 3 isomers. (f) 13 separated from 14, but stereoisomers of 14 could not be separated or assigned.

The rearranged imine 5 should also afford the 2-azapentadienyl anion 3 upon transmetalation. Thus, addition of 5 and $E$-stilbene to $n$-BuLi followed by MeI quench afforded a mixture of stereoisomers of $6 / 7$ plus a new isomer isomer with a Z-propenyl substituent, but in only $22 \%$ yield.

Oxidative cleavage of the alkenyl group was explored, as shown in Schemes 2 and 3. Direct cleavage of the double bond of the diphenyl-substituted 2-propenylpyrrolidines $6 / 7$ or their ammonium salts was not clean using ozonolysis or one-pot oxidations with $\mathrm{RuO}_{4} / \mathrm{NaIO}_{4}$, OsO4/NaIO4, or OsO4/Jones reagent, ${ }^{14}$ presumably because of the presence of the basic nitrogen. Dihydroxylation of $8 / 9$ to the diol 16 followed by periodate cleavage gave the aldehyde 17 in good yield (Scheme 2). Direct conversion of $8 / 9$ to the ester 18 was accomplished using Marshall's recent method. ${ }^{15}$ Oxidative cleavage of the alkenyl group in the phenylthio-substituted 2-propenylpyrrolidines (Scheme 3) was not efficient unless the nitrogen was deactivated and the sulfide was first oxidized to the sulfone. Tosylation of 13/14 gave 19, which was oxidized to the sulfone 20 with Jones' reagent. Marshall's ozonolysis conditions gave the ester 21. Diols such as 16 are reminiscent of biologically active aza-sugars. The preparation of 17, 18, and 21 represents a new approach to the synthesis of novel proline analogues.

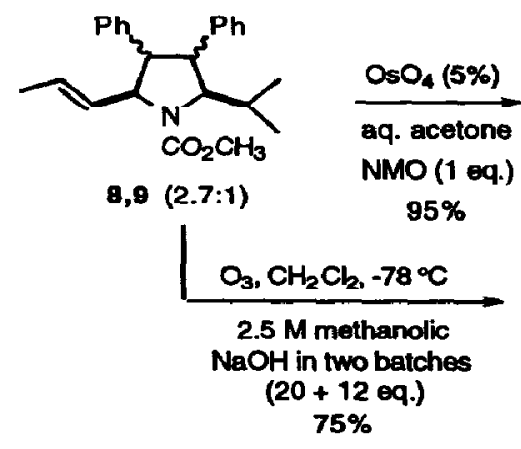

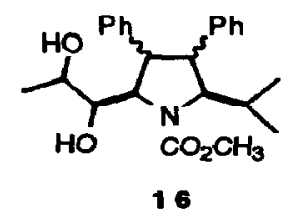

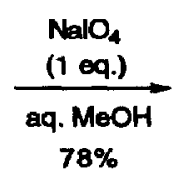<smiles>COC(=O)[C@H]1[C@@H](c2ccccc2)[C@H](c2ccccc2)[C@H](C(C)C)N1C(C)=O</smiles>

18 (2.3:1)

Scheme 2. Oxidative Transformations of 3,4-Dipheny-2-propenylpyrrolidines 


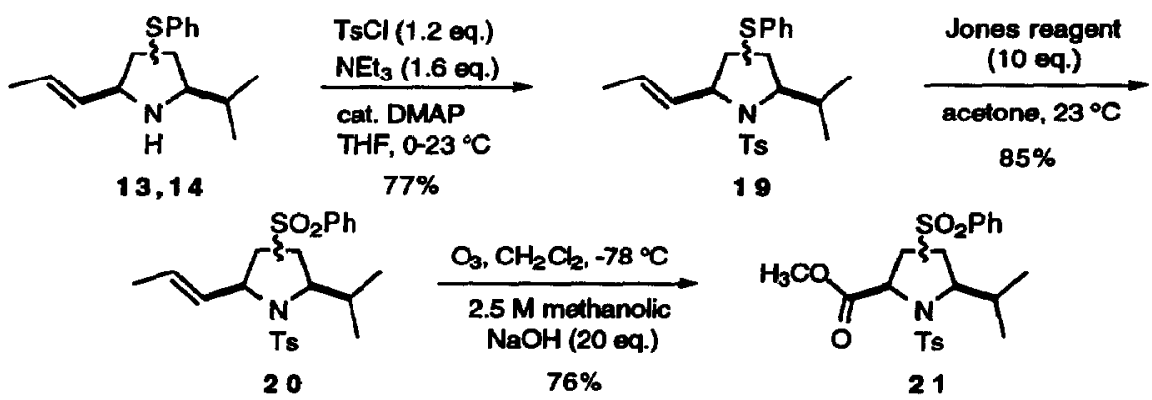

Schame 3. Oxidative Transformations of Phenylthio-Substituted 2-Propenylpyrrolidines

Acknowledgment is made to the donors of the Petroleum Research Fund, administered by the American Chemical Society, for support of this research.

\section{REFERENCES AND NOTES}

(1) (a) Pearson, W. H.; Szura, D. P.; Harter, W. G. Tetrahedron Lett 1988, 29, 761-764. (b) Pearson, W. H.; Postich, M. J. J. Org. Chem. 1992, 57, 6354-6357. (c) Pearson, W. H.; Szura, D. P.; Postich, M. J. J. Am. Chem Soc. 1992, 114, 1329-1345.

(2) For a review of 2-azallyl anion chemistry, see: Kauffmann, T. Angew. Chem, Int. Ed. Engl. 1974, 13, 627-639.

(3) Pearson, W. H.; Stevens, E. P. Tetrahedron Lett. 1994, 35, 2641-2644.

(4) (a) Lown, W. J. In 1,3-Dipolar Cycloaddition Chemistry; A. Padwa, Ed.; Wiley: New York, 1984 ; Vol. 1; pp 663-732. (b) Pearson, W. H. In Studies in Natural Products Chemistry; A. Rahman, Ed; Elsevier: Amsterdam, 1988; Vol. 1; pp 323-358. (c) Vedejs, E. In Advances in Cycloaddition; D. P. Curran, Ed.; JAI Press: Greenwich, CT, 1988; Vol. 1; pp 33-51. (d) Tsuge, O.; Kanemasa, S. Adv. Het. Chem. 1989, 45, 231-349. For a review of intramolecular dipolar cycloadditions, including azomethine ylides, see: (e) Wade, P. A. In Comprehensive Organic Synthesis; B. M. Trost and I. Flcming, Ed.; Pergamon: Oxford, 1991; Vol. 4; pp 1111-1168.

(5) For a recent report of the generation of an alkenyl-substituted azomethine ylide and its cycloaddition with acrylonitrile to form a 2-alkenylpyrrolidine, see: (a) Bourhis, M.; Vercauteren, J. Tetrahedron Lett. 1994, 35, 1981-1984. See also: (b) Fang, J.-M.; Yang, C.-C.; Wang, Y.-W. J. Org. Chem 1989, 54, 481-484.

(6) Hunter, D. H.; Steiner, R. P. Can. J. Chem. 1975, 53, 355-365.

(7) (a) Wolf, G.; Würthwein, E.-U. Chem. Ber. 1991, 124, 655-663. (b) Wolf, G.; Würthwein, E.-U. Tetrahedron Lett. 1988, 29, 3647-3650. See also references to earlier computational studies cited therein.

(8) Epifani, E; Florio, S.; Perrone, D.; Valle, G. Tetrahedron 1991, 47, 4465-4476.

(9) (a) Speckamp, W. N.; Veenstra, S. J.; Dijkink, J.; Fortgens, R. J. Am. Chem. Soc. 1981, 103, 46434645. (b) Speckamp, W. N. Heterocycles 1984, 21, 211-234.

(10) (a) Pearson, W. H. Tetrahedron Lett. 1985, 26, 3527-3530 and references cited therein. See also: (b) Hudlicky, T.; Seoane, G.; Lovelace, T. C. J. Org. Chem. 1988, 53, 2094-2099.

(11) Pearson, W. H.; Postich, M. J. J. Org. Chem. 1992, 57, 6354-6357.

(12) Chong, J. M.; Park, S. B. J. Org. Chem 1992, 57, 2220-2222.

(13) (a) Hails, M. J.; Mann, B. E.; Spencer, C. M. J. Chem. Sac., Dalton Trans. 1983, 729-732. (b) Naruta, Y.; Nishigaichi, Y.; Maruyama, K. Tetrahedron 1989, 45, 1067-1078 and references cited therein.

(14) Henry, J. R.; Weinreb, S. M. J. Org. Chem 1993, 58, 4745.

(15) Marshall, J. A.; Garofalo, A. W. J. Org. Chem 1993, 58, 3675-3680. 